Article

\title{
In Silico Screening Identifies a Novel Potential PARP1 Inhibitor Targeting Synthetic Lethality in Cancer Treatment
}

\author{
Jian Li ${ }^{1, *,+}$, Nan Zhou ${ }^{2, \dagger}$, Peiling Cai ${ }^{1}$ and Jinku Bao ${ }^{2, *}$ \\ 1 School of Medicine, Chengdu University, Chengdu 610106, China; caipeiling@cdu.edu.cn \\ 2 College of Life Sciences and Key Laboratory for Bio-Resources of Ministry of Education, Sichuan University, \\ Chengdu 610064, China; zhnanx@live.com \\ * Correspondence: lijian01@cdu.edu.cn (J.L.); baojinku@scu.edu.cn (J.B.); \\ Tel./Fax: +86-28-8481-4359 (J.L.); +86-28-8541-5171 (J.B.) \\ + These authors contributed equally to this work.
}

Academic Editor: Ge Zhang

Received: 24 December 2015; Accepted: 6 February 2016; Published: 19 February 2016

\begin{abstract}
Synthetic lethality describes situations in which defects in two different genes or pathways together result in cell death. This concept has been applied to drug development for cancer treatment, as represented by Poly (ADP-ribose) polymerase (PARPs) inhibitors. In the current study, we performed a computational screening to discover new PARP inhibitors. Among the 11,247 compounds analyzed, one natural product, ZINC67913374, stood out by its superior performance in the simulation analyses. Compared with the FDA approved PARP1 inhibitor, olaparib, our results demonstrated that the ZINC67913374 compound achieved a better grid score $(-86.8)$ and amber score $(-51.42)$. Molecular dynamics simulations suggested that the PARP1-ZINC67913374 complex was more stable than olaparib. The binding free energy for ZINC67913374 was $-177.28 \mathrm{~kJ} / \mathrm{mol}$ while that of olaparib was $-159.16 \mathrm{~kJ} / \mathrm{mol}$. These results indicated ZINC67913374 bound to PARP1 with a higher affinity, which suggest ZINC67913374 has promising potential for cancer drug development.
\end{abstract}

Keywords: synthetic lethality; PARP inhibitor; DNA damage response (DDR)

\section{Introduction}

The concept of synthetic lethality has recently emerged in the field of cancer treatment. This concept was borrowed from classical genetics to describe situations in which defects in two different genes or pathways together result in cell death, while a defect in one of the two does not affect viability $[1,2]$. Because the deficiency of certain DNA damage response (DDR) gene(s) or pathway(s) are observed in virtually all types of cancer, drugs targeting the complementary pathway of the defective DDR function would be an ideal strategy for cancer treatment with desired high specificity [3].

Poly (ADP-ribose) polymerases (PARPs) inhibitors are a group of chemical compounds that are being developed for cancer treatment under the concept of synthetic lethality. PARPs are enzymes that transfer ADP-ribose moieties to a variety of protein substrates [4]. The physiological function of ADP-ribosylation is best characterized in the context of genome stability maintenance, in which ADP-ribose polymers facilitate the recruitment of the proteins to sites of DNA damage [4]. Inhibition of PARP-1 leads to the accumulation of single-strand breaks (SSBs) that are converted to double strand-strand breaks (DSBs) during DNA replication. The generated DSBs can be repaired either by homologous recombination (HR) or non-homologous end joining (NHEJ) [5,6]. The inhibition of PARP has been shown to be synthetically lethal with loss of BRCA1 and BRCA2, which play essential roles 
in HR-mediated DSB repair [7-9]. Moreover, defects in the DNA damage response proteins, such as NBS1, MRE11, ATR, ATM, FANCD2, FANCA, FANCC, Chk1, Chk2, and ERCC1, also confer selective sensitivity to PARP inhibition [10-14].

The therapeutic potential of PARP inhibitors may extend to a larger cohort of patients than initially indicated. It is of great significance to identify potent PARP1 inhibitors to target the complementary pathways in these synthetic lethal pairs. In the current study, we performed a computational screening to discover new PARP inhibitors with drug development potential. Among the 11,247 compounds analyzed, one natural product, ZINC67913374, was identified as a potential PARP1 inhibitor, because of its superior performance in the simulation analyses.

\section{Results}

\subsection{Docking Performance}

The receiver operating characteristic (ROC) curves for the grid scoring and amber scoring functions were plotted. As shown in Figure 1, the area under curve (AUC) of grid score and amber score were 0.606 and 0.669 , respectively. Both are higher than the random condition, which only gave a 0.500 AUC value.

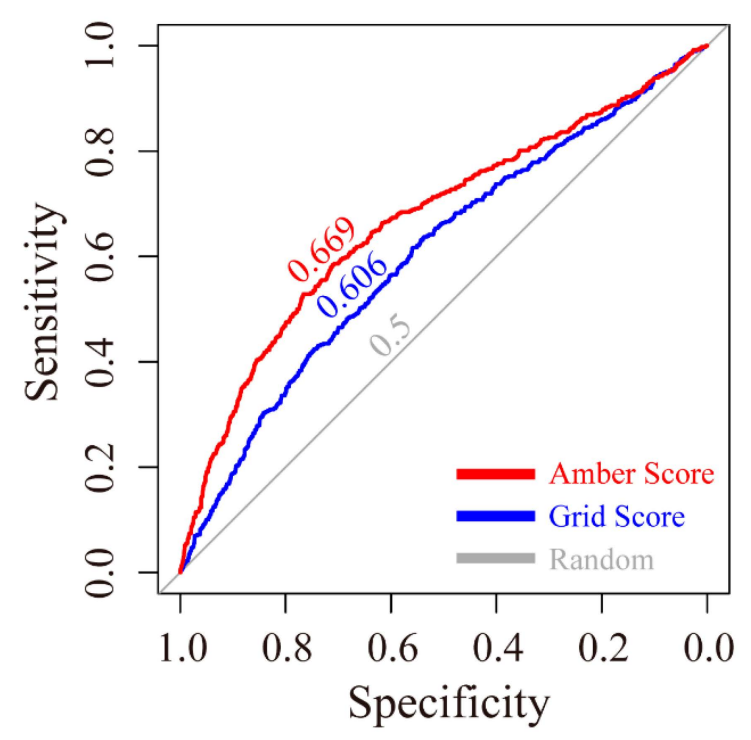

Figure 1. ROC evaluation of grid and amber scoring functions. Corresponding AUC values for each ROC curve are labeled above the line. Color code: Red-amber scoring function; Blue-grid scoring function; Gray-random.

\subsection{Potential PARP1 Inhibitor}

The reference drug, olaparib, achieved a grid score of -61.41 and amber score of -51.18 (Table 1), which was set as the cut-off values for selecting potential PARP1 inhibitors. Compared to olaparib, 631 out of 11,247 natural compounds received a higher grid score. These hits were rescored using the amber scoring function, and one compound, ZINC67913374, stood out with an amber score of -51.42 (Table 1). ZINC67913374 was therefore identified as a potential inhibitor against PARP1. 
Table 1. Candidate PARP1 inhibitors from virtual screening.

Compound

\subsection{Binding Modes}

As shown in Figure 2, both olaparib and ZINC67913374 bound to PARP1 in its binding pocket. They interact with PARP1 through hydrophobic interaction and hydrogen bonds formation. Olaparib bound to PARP1 by forming three hydrogen bonds (Figure 2a): its $\mathrm{O} 3$ formed two hydrogen bonds with the NE and NH2 of Arg878 at a distance of 3.1 and $2.9 \AA$, respectively; N2 of olaparib formed another hydrogen bond with the O of Gly863 at a $2.8 \AA$ distance.

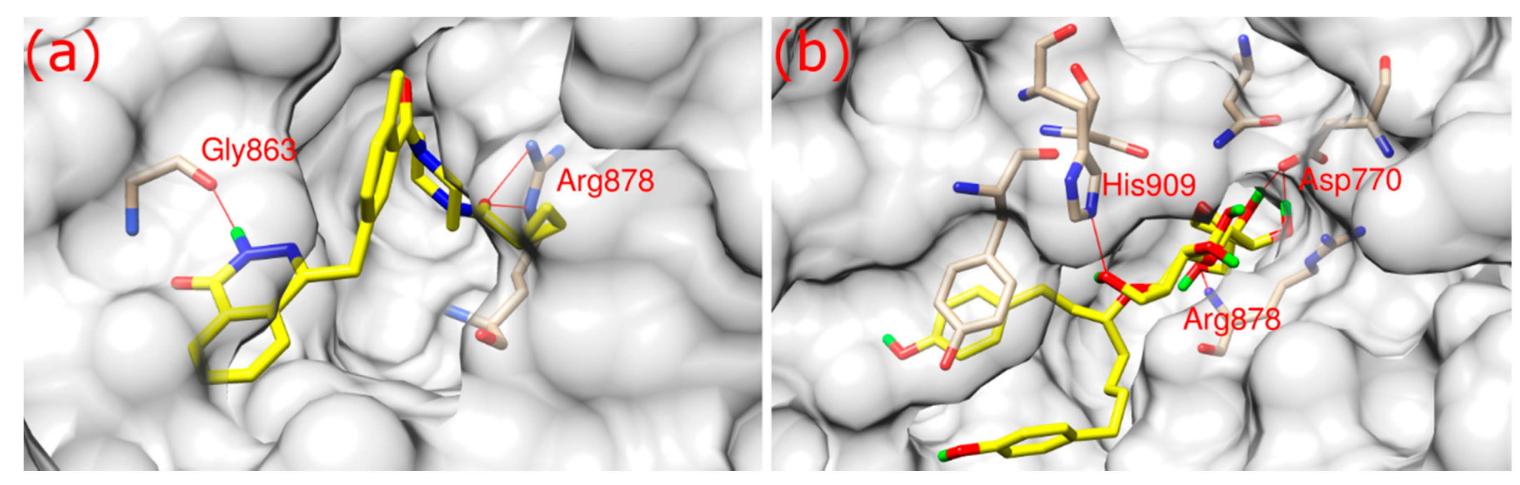

Figure 2. Binding modes of inhibitors towards PARP1 at the binding site. (a) Binding mode of olaparib; (b) binding mode of ZINC67913374. The surface of PARP1 is presented as gray with 70\% transparency. Inhibitors are shown as yellow stick. Corresponding residues of PRAP1 forming hydrogen bonds with ligands are displayed as tan stick. Color code for elements: tan-C of PARP1; yellow $-\mathrm{C}$ of inhibitor; blue- $\mathrm{N}$; red-O; green- $\mathrm{H}$.

ZINC67913374 formed four hydrogen bonds with PARP1 (Figure 2b). Similar to olaparib, the O9 of ZINC67913374 formed one hydrogen bond with the N of Arg878 at a distance of $3 \AA$. ZINC67913374 formed another hydrogen bond at $2.8 \AA$ between its O4 and NE2 of His909. OD2 of ZINC67913374 formed two hydrogen bonds with Asp770, one with O10 (2.6 ̊) and another with O11 (2.5 $)$.

\subsection{Stability of Receptor-Ligand Complex}

MD simulation is exploited to evaluate the stability of a protein-ligand system. As shown in Figure 3, the RMSD of olaparib-PARP1 complex has gradually increased to about $0.34 \mathrm{~nm}$ by $7 \mathrm{~ns}$. Afterwards, it plunged to about $0.16 \mathrm{~nm}$ at $8 \mathrm{~ns}$ and then jumped to the highest point $(0.35 \mathrm{~nm})$ at around $10 \mathrm{~ns}$. The RMSD value fluctuated in the following $6 \mathrm{~ns}$ and stabilized at $0.25 \mathrm{~nm}$ by $16 \mathrm{~ns}$. In terms of PARP1-ZINC67913374 complex, the RMSD reached around $0.3 \mathrm{~nm}$ in just $1 \mathrm{~ns}$. After that, it 
plummeted by $2 \mathrm{~ns}$ and then experienced a rise trend till $4 \mathrm{~ns}$. Then, the RMSD fell and fluctuated at around $0.2 \mathrm{~nm}$ over the next $4 \mathrm{~ns}$. It began fluctuating markedly at $8 \mathrm{~ns}$. The equilibrium $(0.2 \mathrm{~nm})$ was reached by $14 \mathrm{~ns}$, which lasted for $6 \mathrm{~ns}$ until the end of the simulation.

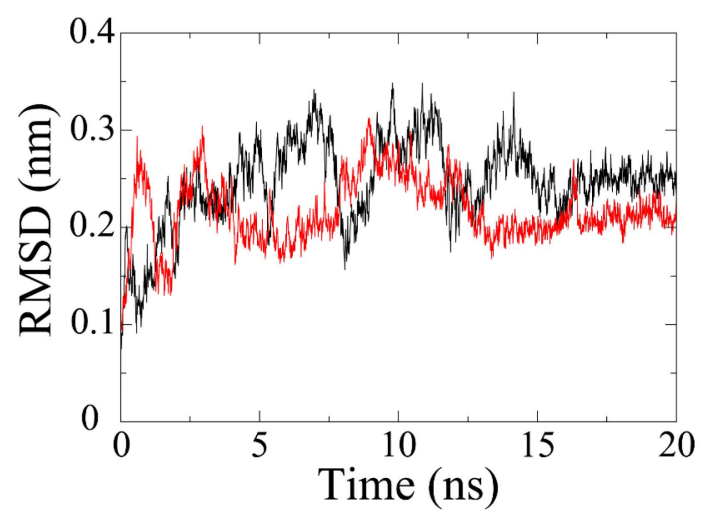

Figure 3. Backbone RMSD of PARP $1{ }^{\circledR} \mathrm{C}$ inhibitor complexes. Black line denotes RMSD of the olaparib system while red line represents the PARP1-ZINC67913374 complex.

\subsection{Binding Free Energy}

The binding free energy of ZINC67913374 and olaparib towards PARP1 were listed in Table 2. Compared with olaparib $(-159.16 \mathrm{~kJ} / \mathrm{mol})$, ZINC67913374 $(-177.28 \mathrm{~kJ} / \mathrm{mol})$ had a smaller binding energy value. For both inhibitors, their gas-phase contribution, namely the combination of van der Waals energy and electrostatic energy, was favorable for inhibitor binding. Their solvation contribution was positive and unfavorable, which may result from strong unfavorable polar energy and weak favorable nonpolar energy. After scrutinizing the four contributing factors, we found both inhibitors had similar van der Waals energy $(-293.07 \mathrm{~kJ} / \mathrm{mol}$ for ZINC67913374 and $-210.36 \mathrm{~kJ} / \mathrm{mol}$ for olaparib) and nonpolar contribution ( $-29.22 \mathrm{~kJ} / \mathrm{mol}$ for ZINC67913374 and $-21.09 \mathrm{~kJ} / \mathrm{mol}$ for olaparib). However, remarkable differences were observed in electrostatic energy $(-327.42 \mathrm{~kJ} / \mathrm{mol}$ for ZINC67913374 and $-89.19 \mathrm{~kJ} / \mathrm{mol}$ for olaparib) and polar solvation energy $(472.47 \mathrm{~kJ} / \mathrm{mol}$ for ZINC67913374 and $161.49 \mathrm{~kJ} / \mathrm{mol}$ for olaparib).

Table 2. Binding free energy $(\mathrm{kJ} / \mathrm{mol})$ of the potential PARP1 inhibitor and olaparib.

\begin{tabular}{ccc}
\hline Inhibitor Components $^{\text {a }}$ & ZINC67913374 & Olaparib \\
\hline$\Delta \mathrm{E}_{\mathrm{vdw}}$ & $-293.07 \pm 10.25$ & $-210.36 \pm 11.77$ \\
$\Delta \mathrm{E}_{\text {ele }}$ & $-327.42 \pm 27.34$ & $-89.19 \pm 15.22$ \\
$\Delta \mathrm{G}_{\text {ploar }}$ & $472.47 \pm 28.21$ & $161.49 \pm 13.88$ \\
$\Delta \mathrm{G}_{\text {nonpolar }}$ & $-29.22 \pm 0.92$ & $-21.09 \pm 0.63$ \\
$\Delta \mathrm{G}_{\text {bind }}$ & $-177.24 \pm 24.78$ & $-159.16 \pm 15.13$ \\
\hline
\end{tabular}

a $\Delta \mathrm{E}_{\mathrm{vdw}}$, van der Waals energy; $\Delta \mathrm{E}_{\text {ele }}$, electrostatic contribution; gas-phase energy consists of $\Delta \mathrm{E}_{\mathrm{vdw}}$ and $\Delta \mathrm{E}_{\text {ele }}$; $\Delta \mathrm{G}_{\text {polar }}$, polar solvation energy; $\Delta \mathrm{G}_{\text {nonpolar }}$, nonpolar solvation energy; the solvation free energy is a sum of $\Delta \mathrm{G}_{\text {polar }}$ and $\Delta \mathrm{G}_{\text {nonpolar }} ; \Delta \mathrm{G}_{\text {bind }}$, binding energy; $\Delta \mathrm{G}_{\text {bind }}=\Delta \mathrm{E}_{\mathrm{vdw}}+\Delta \mathrm{E}_{\text {ele }}+\Delta \mathrm{G}_{\text {polar }}+\Delta \mathrm{G}_{\text {nonpolar }}$.

The 2D interaction diagrams generated by LigPlot+ for olaparib- and ZINC67913374-PARP1 complexes were presented in Figure 4 [15]. The two complexes are fairly similar, so that they can be superimposed on each other. The superposition of the two related diagrams highlighted conserved interactions within olaparib- and ZINC67913374-PARP1 complexes. As shown in Figure 4, His862, Tyr907, Tyr896, Aan868, Arg878, Asp766, and Gly863 were conserved residues. Gly863 and Tyr907 had been reported as key amino acid residues for inhibitors-PARP1 interactions [16]. Gly863 participated in hydrogen bonding interaction network, while Tyr907 is involved in pi-pi stacking. Tyr907 was a key residue, which was not identified in olaparib-PARP1 interaction, but in the 
interaction between ZINC67913374 and PARP1. The other residues, including Ser904, Phe897, Ala898, Glu763, Leu877, Ile872, Met890, and Gly888, interacted with PARP1 via hydrophobic contacts. All the data above supported a stronger interaction between ZINC67913374 and PARP1, compared with the olaparib-PARP1 interaction.
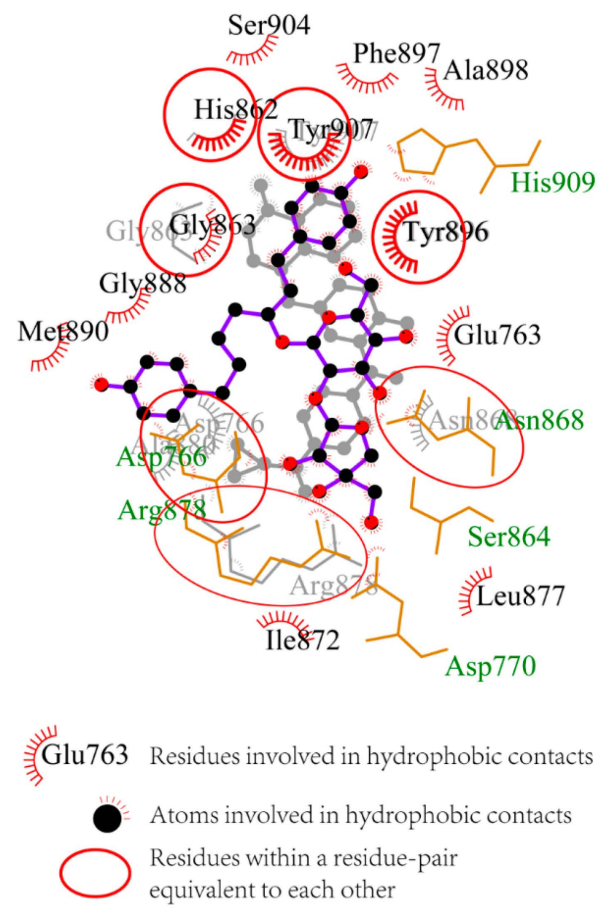

Figure 4. Superimposed 2D interaction diagrams of olaparib (background) and ZINC67913374 (foreground) with PARP1. Ball and stick denotes ligands. Corresponding PARP1 residues are shown as wires.

Backbone RMSF (root mean square fluctuation) of PARP1 within ZINC67913374-PARP1 complex was comparable to that of the olaparib-PARP1 system (Figure 5). We scrutinized the contribution of each residue in the receptor-ligand interaction by binding energy decomposition (Figure 6). In both PARP1-inhibitor complexes, Glu763, Asp766, Tyr896, Ser904, and Tyr907 were critical residues for the binding interaction. This observation was consistent with the result that ZINC67913374 and olaparib share similar features on the RMSF profile when binding to PARP1.

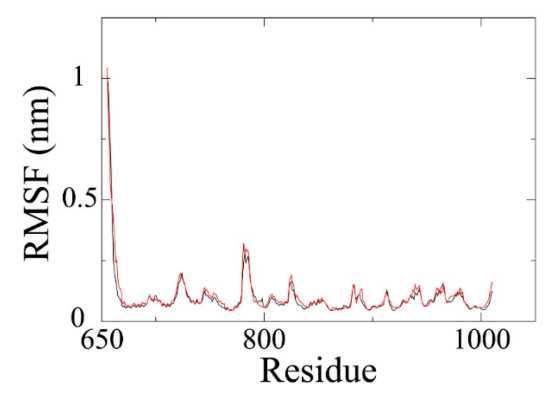

Figure 5. RMSF plots of backbone atoms for PARP $1{ }^{\circledR}$ Cinhibitor systems. Black line is for olaparib and red line is the PARP1-ZINC67913374 system. 


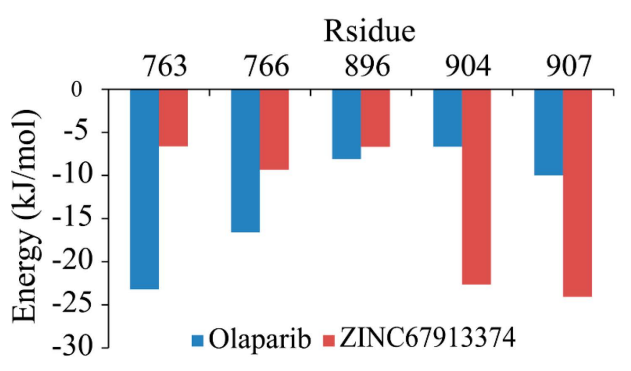

Figure 6. Binding free energy decomposition on a per-residue basis for olaparib- and ZINC67913374-PARP1 complexes.

\subsection{ADMET Analysis}

We predicted ZINC67913374's ADMET using admetSAR, a free tool for evaluating chemical ADMET properties [17]. The result (Table A1) shows even with $>500$ molecular weight, ZINC67913374 can permeate the blood-brain barrier. The prediction also shows it has no AMES toxicity and no carcinogenicity.

\section{Discussion}

Currently, the majority of cancer therapies target proliferating cells rather than cancer cells per se. Cancer cells with a low proliferative index may evade treatment, while highly proliferative normal cells are also attacked by the same therapies. Taking advantage of the dysregulated DNA damage response in cancer using the synthetic lethality approach may be one of the most promising prospects for the future of cancer treatment. Similar to ovarian and breast cancers, some sporadic prostate, pancreatic and other tumors also possess DNA damage response defects due to mutation or epigenetic inactivation of HR components, suggesting that PARP inhibitors might be more broadly applicable.

By computational approaches integrating virtual screening, molecular dynamics simulation, binding free energy calculation and decomposition, we discovered a new potential inhibitor for PARP1 from natural products. Compared to the currently FDA-approved olaparib, this chemical exhibited a higher binding affinity to PARP1. It is projected to result in dysregulation of DNA damage repair, indicating its therapeutic potential for the treatment of cancers. Our results demonstrated that ZINC67913374 has promising potential as a PARP1 inhibitor for cancer drug development. Song and colleagues described the identification of four PARP1 inhibitors from a large number of natural products by in silico screening and in vitro enzymatic assay [18]. The screening performed in the current study focused on the AnalytiCon Discovery NP database. This database has a high percentage of unique compounds compared with other databases, which should theoretically increase the chance for discovering new potential PARP inhibitors. Certainly, the physiological activity of this compound needs to be validated with in vitro and in vivo studies in the next stage of the study. On the other hand, it is imperative to report this intriguing result of the researchers work in the field. A greater understanding of the basis of PARP inhibitor response is required for translational and clinical development of these agents, and in order to establish which patients may derive the most therapeutic benefit from this class of inhibitors.

\section{Materials and Methods}

\subsection{Structure Preparation}

The 3D structure of PARP1 was downloaded from the RCSB Protein Data Bank (PDB) using accession number 4UND [19], in which PARP1 is in complex with a known inhibitor named BMN673.This structure is used for identifying the binding site of PARP1. The molecular graphics of PARP1 were prepared and analyzed with the UCSF Chimera package [20]. In this process, (i) solvent 
and non-complexed ions were removed from PARP1; and (ii) hydrogens and charges (of amber ff99sb force field) were added to the protein.

The molecule library of natural compounds from AnalytiCon Discovery NP, containing 11,247 ZINC entries, was downloaded on 25 July 2015 from the ZINC database [21]. All these natural products have been filtered according to the criteria from ZINC and are provided in ready-to-dock, 3D formats.

On 19 December 2014, the U.S. Food and Drug Administration approved olaparib capsules (Lynparza, AstraZeneca Pharmaceuticals LP) as a monotherapy for advanced ovarian cancer after treatment with three or more prior lines of chemotherapy. The FDA approved olaparib was used as a reference drug for selecting hits and candidate PARP1 inhibitors.

\subsection{Dock and Virtual Screening}

For the sake of discovering new PARP1 inhibitors with drug development potential, a virtual screening was carried out by docking natural compounds to the binding site of PARP1 using UCSF DOCK 6 [22]. The known inhibitor, olaparib, was used as a reference drug to select hits. The DOCK suite of programs first docked all the natural compounds to PARP1 by assigning them grid scores that represent to what extent a given ligand would bind to a specific target. The scores were compared with that of olaparib. The compounds with higher scores were selected as hits for the second round of screening. Subsequently, the hit compounds were rescored by DOCK amber rescoring function, which allows small structural rearrangements to reproduce the so-called induced fit while performing docking. The compounds achieved higher amber scores than that of olaparib were chosen as candidate PARP1 inhibitors.

The specific running parameters used in our study for DOCK were: (1) change probe radius was 1.0; (2) maximum sphere radius was $3.0 \AA$; (3) minimum sphere radius was $1.0 \AA$; (4) the radius used to select binding site from the position where the known olaparib bound to was $8.0 \AA$; and (5) extra margin enclosed in all 6 directions was 3.0.

\subsection{Dock Protocol Evaluation}

The ROC curve was employed to illustrate the performance of DOCK. For binary classification, the possible outcomes fall into four categories: true positive (TP, a prediction is positive and the actual value is also positive), false positive (FP, a prediction is positive but the actual value is negative), true negative (TN, both the prediction outcome and actual value are negative), and false negative (FN, the prediction outcome is negative while the actual value is positive). The true positive rate (TPR) known as sensitivity can be expressed as [23]

$$
\mathrm{TPR}=\text { sensitivity }=\mathrm{TP} /(\mathrm{TP}+\mathrm{FN})
$$

The false positive rate (FPR) is also known as the fall-out and can be calculated as

$$
\mathrm{FPR}=1-\text { specificity }=1-\mathrm{TN} /(\mathrm{TN}+\mathrm{FP})
$$

At the beginning, actives (positive PARP1 inhibitors) and decoys (negative PARP1 inhibitors) were downloaded (on 25 July 2015) from the DUD-E database, which provides active compounds and challenging decoys for molecular docking programs [24]. 742 actives and 3710 decoys were docked to the binding site of PARP1. These compounds were sequentially analyzed by grid scoring and amber scoring. pROC library within R was used to plot ROC curves (by plotting the sensitivity against the specificity at various threshold settings) and calculate values of area under the ROC curve (AUC), by which the performance of DOCK can be evaluated quantitatively [25]. 


\subsection{Simulation}

MD simulations were performed using GROMACS 4.5 [26] package and amber ff99sb force field [27] with TIP3P water model [28]. Particle Mesh Ewald (PME) [29] was exploited to consider the long-range electrostatic interactions and the Linear Constraint Solver (LINCS) [30] algorithm was used to constrain bonds. The receptor-ligand complexes were solvated in a dodecahedron box of water, with a distance of 1.0 between the solute and the box. All systems were neutralized by adding $\mathrm{Na}^{+}$and $\mathrm{Cl}^{-}$at $0.15 \mathrm{~mol} / \mathrm{L}$. Before MD simulations, the complexes were relaxed to $<1000 \mathrm{~kJ} / \mathrm{mol} / \mathrm{nm}$ by up to 50,000 cycles of steep descent minimization. After energy minimization, temperature of the system was controlled in the NVT (constant number of particles, volume, and temperature) ensemble to $300 \mathrm{~K}$ over 100 ps. The 100 ps NPT (constant number of particles, pressure, and temperature) equilibration was then performed with a reference pressure of 1 bar. After that, $20 \mathrm{~ns}$ MD simulations were performed with a time step of $2 \mathrm{fs}$ and the coordinates of the complexes were saved every 8 ps.

\subsection{Free Energy Calculation and Decomposition}

Molecular mechanics Poisson-Boltzmann surface area (MM-PBSA) was applied as a scoring function in computational drug design to estimate the interaction free energies in biomolecular interactions [31]. The binding free energy of a protein-ligand system in solvent can be given by [32]:

$$
\Delta \mathrm{G}_{\text {bind }}=\mathrm{G}_{\text {complex }}-\left(\mathrm{G}_{\text {protein }}+\mathrm{G}_{\text {ligand }}\right)
$$

where Gcomplex is the total free energy of the protein-ligand complex and Gprotein and Gligand represent free energies of the isolate protein and ligand, respectively.

In this study, the GROMACS tool g_mmpbsa was used to calculate the binding free energy of the protein with ligand [33]. The MM-PBSA approach was used to calculate the binding free energy as follows $[33,34]$ :

$$
\Delta \mathrm{G}_{\text {bind }}=\Delta \mathrm{E}_{\text {gas }}+\Delta \mathrm{G}_{\text {solv }}=\Delta \mathrm{E}_{\mathrm{vdw}}+\Delta \mathrm{E}_{\mathrm{ele}}+\Delta \mathrm{G}_{\text {polar }}+\Delta \mathrm{G}_{\text {nonpolar }}
$$

$\Delta \mathrm{E}_{\text {gas }}$ is the average molecular mechanics potential energy in a vacuum (i.e., gas-phase energy), which includes van der Waals $\left(\Delta \mathrm{E}_{\mathrm{vdw}}\right)$ and electrostatic $\left(\Delta \mathrm{E}_{\text {ele }}\right)$ interactions; $\Delta \mathrm{G}_{\text {solv }}$ denotes contribution to the solvation free energy that consists of polar solvation $\left(\Delta G_{\text {polar }}\right)$ and nonpolar solvation $\left(\Delta G_{\text {nonpolar }}\right)$ energies.

Using the g_mmpbsa, the binding free energy of protein-ligand complex was calculated from 11 snapshots extracted every $0.2 \mathrm{~ns}$ from the 18 to $20 \mathrm{~ns}$ MD trajectory. Furthermore, the binding energy was decomposed on a per residue basis to analyze the individual energy contributions of each residue to the protein-ligand interaction.

\section{Conclusions}

ZINC67913374 identified in this study is a potent PARP1 inhibitor with drug development potential, as reveal by the extensive bioinformatics simulation analyses. The FDA approval of olaparib and other on-going PARP1 inhibition related clinical trials validates synthetic lethality as an effective therapeutic strategy in cancer drug development. The success of PARP1 inhibitors encourages the characterization and targeting of other synthetic lethality pairs in DNA damage response and repair pathways.

Supplementary Materials: Supplementary materials can be found at http://www.mdpi.com/1422-0067/17/2/ 258/s1.

Acknowledgments: We would like to thank Donald Holzschu (Ohio University) and Caroline Tang (Yale University) for language editing. This work was supported by Natural Science Foundation of China (No. 31500657) and Chengdu University New Faculty Start-up funding (No. 2081915019). 
Author Contributions: Jian Li, Nan Zhou and Jinku Bao conceived and designed the experiments; Jian Li, Nan Zhou, and Peiling Cai performed the experiments and analyzed the data; Jian Li, Nan Zhou and Jinku Bao wrote the paper; and all authors read and approved the final manuscript.

Conflicts of Interest: The authors declare no conflict of interest.

\section{Abbreviations}

$\begin{array}{ll}\text { DDR } & \text { DNA damage response } \\ \text { PARP } & \text { Poly (ADP-ribose) polymerase } \\ \text { SSB } & \text { single-strand break } \\ \text { DSB } & \text { double strand-strand break } \\ \text { HR } & \text { homologous recombination } \\ \text { NHEJ } & \text { non-homologous end joining } \\ \text { LINCS } & \text { Linear Constraint Solver } \\ \text { MM-PBSA } & \text { Molecular mechanics Poisson-Boltzmann surface area }\end{array}$

\section{Appendix}

Table A1. ADMET properties of ZINC67913374.

\begin{tabular}{|c|c|c|}
\hline Property & Value & Prabability \\
\hline Blood-Brain Barrier & $\mathrm{BBB}+$ & 0.7028 \\
\hline Human Intestinal Absorption & HIA- & 0.7104 \\
\hline Caco-2 Permeability & Caco2- & 0.824 \\
\hline P-glycoprotein Substrate & Substrate & 0.6847 \\
\hline P-glycoprotein Inhibitor (I) & Non-inhibitor & 0.7057 \\
\hline P-glycoprotein Inhibitor (II) & Non-inhibitor & 0.7339 \\
\hline Renal Organic Cation Transporter & Non-inhibitor & 0.7435 \\
\hline CYP450 2C9 Substrate & Non-substrate & 0.8328 \\
\hline CYP450 2D6 Substrate & Non-substrate & 0.8277 \\
\hline CYP450 3A4 Substrate & Non-substrate & 0.5479 \\
\hline CYP450 1A2 Inhibitor & Non-inhibitor & 0.9058 \\
\hline CYP450 2C9 Inhibitor & Non-inhibitor & 0.8699 \\
\hline CYP450 2D6 Inhibitor & Non-inhibitor & 0.8892 \\
\hline CYP450 2C19 Inhibitor & Non-inhibitor & 0.8244 \\
\hline CYP450 3A4 Inhibitor & Non-inhibitor & 0.9023 \\
\hline CYP Inhibitory Promiscuity & Low CYP Inhibitory Promiscuity & 0.8839 \\
\hline Human ERG Inhibition (I) & Weak inhibitor & 0.8461 \\
\hline Human ERG Inhibition (II) & Inhibitor & 0.5092 \\
\hline AMES Toxicity & Non AMES toxic & 0.9003 \\
\hline Carcinogens & Non-carcinogens & 0.9566 \\
\hline Fish Toxicity & High FHMT & 0.9056 \\
\hline Tetrahymena Pyriformis Toxicity & High TPT & 0.9662 \\
\hline Honey Bee Toxicity & High HBT & 0.6725 \\
\hline Biodegradation & Not ready biodegradable & 0.9337 \\
\hline Acute Oral Toxicity & III & 0.5751 \\
\hline Carcinogenicity (Three-class) & Non-required & 0.5401 \\
\hline Model & Value & Unit \\
\hline Aqueous solubility & -1.3682 & LogS \\
\hline Caco-2 Permeability & -0.5376 & LogPapp, cm/s \\
\hline Rat Acute Toxicity & 2.5494 & $\mathrm{LD} 50, \mathrm{~mol} / \mathrm{kg}$ \\
\hline Fish Toxicity & 1.8239 & $\mathrm{pLC} 50, \mathrm{mg} / \mathrm{L}$ \\
\hline Tetrahymena Pyriformis Toxicity & 0.4575 & pIGC50, ug/L \\
\hline
\end{tabular}




\section{References}

1. Ashworth, A. A synthetic lethal therapeutic approach: Poly(ADP) ribose polymerase inhibitors for the treatment of cancers deficient in DNA double-strand break repair. J. Clin. Oncol. 2008, 26, 3785-3790. [CrossRef] [PubMed]

2. Garber, K. Running interference: Pace picks up on synthetic lethality research. J. Natl. Cancer Inst. 2004, 96, 982-983. [CrossRef] [PubMed]

3. Kaelin, W.G., Jr. The concept of synthetic lethality in the context of anticancer therapy. Nat. Rev. Cancer 2005, 5, 689-698. [CrossRef] [PubMed]

4. Rouleau, M.; Patel, A.; Hendzel, M.J.; Kaufmann, S.H.; Poirier, G.G. PARP inhibition: PARP1 and beyond. Nat. Rev. Cancer 2010, 10, 293-301. [CrossRef] [PubMed]

5. Bryant, H.E.; Petermann, E.; Schultz, N.; Jemth, A.S.; Loseva, O.; Issaeva, N.; Johansson, F.; Fernandez, S.; McGlynn, P.; Helleday, T. PARP is activated at stalled forks to mediate Mre11-dependent replication restart and recombination. EMBO J. 2009, 28, 2601-2615. [CrossRef] [PubMed]

6. De Vos, M.; Schreiber, V.; Dantzer, F. The diverse roles and clinical relevance of PARPs in DNA damage repair: Current state of the art. Biochem. Pharmacol. 2012, 84, 137-146. [CrossRef] [PubMed]

7. Bryant, H.E.; Schultz, N.; Thomas, H.D.; Parker, K.M.; Flower, D.; Lopez, E.; Kyle, S.; Meuth, M.; Curtin, N.J.; Helleday, T. Specific killing of BRCA2-deficient tumours with inhibitors of poly(ADP-ribose) polymerase. Nature 2005, 434, 913-917. [CrossRef] [PubMed]

8. Farmer, H.; McCabe, N.; Lord, C.J.; Tutt, A.N.; Johnson, D.A.; Richardson, T.B.; Santarosa, M.; Dillon, K.J.; Hickson, I.; Knights, C.; et al. Targeting the DNA repair defect in BRCA mutant cells as a therapeutic strategy. Nature 2005, 434, 917-921. [CrossRef] [PubMed]

9. Heyer, W.D.; Ehmsen, K.T.; Liu, J. Regulation of homologous recombination in eukaryotes. Annu. Rev. Genet. 2010, 44, 113-139. [CrossRef] [PubMed]

10. Gilardini Montani, M.S.; Prodosmo, A.; Stagni, V.; Merli, D.; Monteonofrio, L.; Gatti, V.; Gentileschi, M.P.; Barilà, D.; Soddu, S. ATM-depletion in breast cancer cells confers sensitivity to PARP inhibition. J. Exp. Clin. Cancer Res. 2013, 32, 95. [CrossRef] [PubMed]

11. Ihnen, M.; zu Eulenburg, C.; Kolarova, T.; Qi, J.W.; Manivong, K.; Chalukya, M.; Dering, J.; Anderson, L.; Ginther, C.; Meuter, A.; et al. Therapeutic potential of the poly(ADP-ribose) polymerase inhibitor rucaparib for the treatment of sporadic human ovarian cancer. Mol. Cancer Ther. 2013, 12, 1002-1015. [CrossRef] [PubMed]

12. McCabe, N.; Turner, N.C.; Lord, C.J.; Kluzek, K.; Bialkowska, A.; Swift, S.; Giavara, S.; O'Connor, M.J.; Tutt, A.N.; Zdzienicka, M.Z.; et al. Deficiency in the repair of DNA damage by homologous recombination and sensitivity to poly(ADP-ribose) polymerase inhibition. Cancer Res. 2006, 66, 8109-8115. [CrossRef] [PubMed]

13. Postel-Vinay, S.; Bajrami, I.; Friboulet, L.; Elliott, R.; Fontebasso, Y.; Dorvault, N.; Olaussen, K.A.; André, F.; Soria, J.C.; Lord, C.J.; et al. A high-throughput screen identifies PARP1/2 inhibitors as a potential therapy for ERCC1-deficient non-small cell lung cancer. Oncogene 2013, 32, 5377-5387. [CrossRef] [PubMed]

14. Williamson, C.T.; Kubota, E.; Hamill, J.D.; Klimowicz, A.; Ye, R.; Muzik, H.; Dean, M.; Tu, L.; Gilley, D.; Magliocco, A.M.; et al. Enhanced cytotoxicity of PARP inhibition in mantle cell lymphoma harbouring mutations in both ATM and p53. EMBO Mol. Med. 2012, 4, 515-527. [CrossRef] [PubMed]

15. Laskowski, R.A.; Swindells, M.B. LigPlot+: Multiple ligand-protein interaction diagrams for drug discovery. J. Chem. Inf. Model. 2011, 51, 2778-2786. [CrossRef] [PubMed]

16. Ferraris, D.V. Evolution of poly(ADP-ribose) polymerase-1 (PARP-1) inhibitors. From concept to clinic. J. Med. Chem. 2010, 53, 4561-4584. [CrossRef] [PubMed]

17. Cheng, F.; Li, W.; Zhou, Y.; Shen, J.; Wu, Z.; Liu, G.; Lee, P.W.; Tang, Y. admetSAR: A comprehensive source and free tool for assessment of chemical ADMET properties. J. Chem. Inf. Model. 2012, 52, 3099-3105. [CrossRef] [PubMed]

18. Song, M.; Li, J.L.; Li, X.P.; Kan, S.F. Targeting human poly(ADP-Ribose) polymerase-1 with natural medicines and its potential applications in ovarian cancer therapeutics. Arch. Pharm. 2015. [CrossRef] [PubMed]

19. Berman, H.M.; Westbrook, J.; Feng, Z.; Gilliland, G.; Bhat, T.N.; Weissig, H.; Shindyalov, I.N.; Bourne, P.E. The protein data bank. Nucleic Acids Res. 2000, 28, 235-242. [CrossRef] [PubMed] 
20. Pettersen, E.F.; Goddard, T.D.; Huang, C.C.; Couch, G.S.; Greenblatt, D.M.; Meng, E.C.; Ferrin, T.E. UCSF Chimera-A visualization system for exploratory research and analysis. J. Comput. Chem. 2004, 25, 1605-1612. [CrossRef] [PubMed]

21. Irwin, J.J.; Sterling, T.; Mysinger, M.M.; Bolstad, E.S.; Coleman, R.G. ZINC: A free tool to discover chemistry for biology. J. Chem. Inf. Model. 2012, 52, 1757-1768. [CrossRef] [PubMed]

22. Allen, W.J.; Balius, T.E.; Mukherjee, S.; Brozell, S.R.; Moustakas, D.T.; Lang, P.T.; Case, D.A.; Kuntz, I.D.; Rizzo, R.C. DOCK 6: Impact of new features and current docking performance. J. Comput. Chem. 2015, 36, 1132-1156. [CrossRef] [PubMed]

23. Zhou, N.; Zhang, J.; Feng, L.; Lu, B.; Wang, Z.; Sun, R.; Wu, C.; Bao, J. IntApop: A web service for predicting apoptotic protein interactions in humans. Biosystems 2013, 114, 238-244. [CrossRef] [PubMed]

24. Mysinger, M.M.; Carchia, M.; Irwin, J.J.; Shoichet, B.K. Directory of useful decoys, enhanced (DUD-E): Better ligands and decoys for better benchmarking. J. Med. Chem. 2012, 55, 6582-6594. [CrossRef] [PubMed]

25. Robin, X.; Turck, N.; Hainard, A.; Tiberti, N.; Lisacek, F.; Sanchez, J.; Müller, M. pROC: An open-source package for $\mathrm{R}$ and $\mathrm{S}_{+}$to analyze and compare ROC curves. BMC Bioinform. 2011, 12, 77. [CrossRef] [PubMed]

26. Pronk, S.; Páll, S.; Schulz, R.; Larsson, P.; Bjelkmar, P.; Apostolov, R.; Shirts, M.R.; Smith, J.C.; Kasson, P.M.; van der Spoel, D.; et al. GROMACS 4.5: A high-throughput and highly parallel open source molecular simulation toolkit. Bioinformatics 2013, 29, 845-854. [CrossRef] [PubMed]

27. Li, J.; Zhou, N.; Liu, W.; Li, J.; Feng, Y.; Wang, X.; Wu, C.; Bao, J. Discover natural compounds as potential phosphodiesterase-4B inhibitors via computational approaches. J. Biomol. Struct. Dyn. 2015, 1-60. [CrossRef] [PubMed]

28. Jorgensen, W.L.; Chandrasekhar, J.; Madura, J.D.; Impey, R.W.; Klein, M.L. Comparison of simple potential functions for simulating liquid water. J. Chem. Phys. 1983, 79, 926-935. [CrossRef]

29. Darden, T.; York, D.; Pedersen, L. Particle mesh Ewald: An $N \cdot \log N$ method for Ewald sums in large systems. J. Chem. Phys. 1993, 98, 10089-10092. [CrossRef]

30. Hess, B. P-LINCS: A parallel linear constraint solver for molecular simulation. J. Chem. Theory Comput. 2008, 4, 116-122. [CrossRef] [PubMed]

31. Homeyer, N.; Gohlke, H. Free energy calculations by the molecular mechanics poisson-boltzmann surface area method. Mol. Inform. 2012, 31, 114-122. [CrossRef]

32. Kollman, P.A.; Massova, I.; Reyes, C.; Kuhn, B.; Huo, S.; Chong, L.; Lee, M.; Lee, T.; Duan, Y.; Wang, W.; et al. Calculating structures and free energies of complex molecules: combining molecular mechanics and continuum models. Acc. Chem. Res. 2000, 33, 889-897. [CrossRef] [PubMed]

33. Kumari, R.; Kumar, R.; Lynn, A. g_mmpbsa-A GROMACS Tool for High-Throughput MM-PBSA Calculations. J. Chem. Inf. Model. 2014, 54, 1951-1962. [CrossRef] [PubMed]

34. Li, J.; Zhou, N.; Luo, K.; Zhang, W.; Li, X.; Wu, C.; Bao, J. In silico discovery of potential VEGFR-2 inhibitors from natural derivatives for anti-angiogenesis therapy. Int. J. Mol. Sci. 2014, 15, 15994-16011. [CrossRef] [PubMed]

(C) 2016 by the authors; licensee MDPI, Basel, Switzerland. This article is an open access article distributed under the terms and conditions of the Creative Commons by Attribution (CC-BY) license (http://creativecommons.org/licenses/by/4.0/). 